\title{
Nonadaptive radiation of the gut microbiome in an adaptive radiation of Cyprinodon pupfishes with minor shifts for scale-eating
}

\author{
Joseph Heras ${ }^{1}$ and Christopher Martin ${ }^{2}$ \\ ${ }^{1}$ California State University San Bernardino \\ ${ }^{2}$ University of California Berkeley
}

January 26, 2021

\begin{abstract}
Adaptive radiations offer an excellent opportunity to understand the eco-evolutionary dynamics of gut microbiota and host niche specialization. In a laboratory common garden, we compared the gut microbiota of two novel trophic specialists, a scaleeater and a molluscivore, to a set of four outgroup generalist populations from which this adaptive radiation originated. We predicted an adaptive and highly divergent microbiome composition in the specialists matching their rapid rates of craniofacial diversification in the past 10 kya. We measured gut lengths and sequenced 16S rRNA amplicons of gut microbiomes from lab-reared fish fed the same high protein diet for one month. In contrast to our predictions, gut microbiota largely reflected 5 Mya phylogenetic divergence times among generalist populations in support of phylosymbiosis. However, we did find significant enrichment of Burkholderiaceae bacteria in both lab-reared scale-eater populations. These bacteria sometimes digest collagen, the major component of fish scales, supporting an adaptive shift. We also found some enrichment of Rhodobacteraceae and Planctomycetacia in lab-reared molluscivore populations, but these bacteria target cellulose. Minor shifts in gut microbiota appear adaptive for scale-eating in this radiation, whereas overall microbiome composition was phylogenetically conserved. This contrasts with predictions of adaptive radiation theory and observations of rapid diversification in all other trophic traits in these hosts, including craniofacial morphology, foraging behavior, aggression, and gene expression, suggesting that microbiome divergence proceeds as a nonadaptive radiation.
\end{abstract}

\section{Introduction}

Rapid evolutionary change can alter ecological processes which in turn change the course of evolutionary processes (Turcotte et al. 2013; Matthews et al. 2016). This process is described as eco-evolutionary dynamics, which provides a framework for understanding the interplay between evolution and ecological interactions (Rudman et al. 2018; Post et al. 2009). The emergence of studies that focus on eco-evolutionary dynamics has provided more insight on the processes of community assembly, ecological speciation, and adaptive radiations (Rudman et al. 2018). A better understanding of these eco-evolutionary dynamics can be applied to host-microbiota interactions, in which the co-evolutionary processes of the microbiome can impact host performance and fitness (Gould et al. 2018; Macke et al. 2017; Walters et al. 2020). The microbial community may also play a large role in the physiology, ecology, and evolution of the host (Baldo et al. 2017; Trevelline and Kohl 2020).

Several studies have now examined gut microbiome diversification in an adaptive radiation of hosts, including fishes (Baldo et al. 2017; Baldo et al. 2019; Loo et al. 2019; Macke et al. 2017; Rennison et al. 2019). Phylosymbiosis, in which the host microbiome recapitulates host phylogeny, is frequently the primary hypothesis in these studies (Brooks et al. 2016; Lim and Bordenstein, 2020). However, these studies rarely examine outgroups to the focal radiation in order to compare rates of microbiome divergence. Furthermore, phylosymbiosis (comparable to phylogenetic conservatism; Losos, 2008) is actually the antithesis to the theory of adaptive radiation, which predicts that the microbiome within an adaptive radiation should 
diverge far more quickly than outgroup taxa due to rapid ecological divergence and specialization (Stroud and Losos 2016, Schluter 2000, Martin and Richards 2019, Gillespie et al. 2020, Rundell and Price 2009). Thus, we predicted greater microbiome divergence within a recent adaptive radiation of trophic specialists than in outgroup generalist taxa with far older divergence times (5 Mya), in contrast to the predictions of phylosymbiosis.

An adaptive radiation of Cyprinodon pupfishes provides an excellent opportunity to test the relative roles of rapid trophic divergence and phylosymbiosis in shaping the gut microbiome. Pupfishes are found in saline lakes or coastal areas throughout the Caribbean and Atlantic (most are allopatric) and within isolated desert pools and streams (Martin et al. 2016, 2020; Echelle and Echelle 2020). However, there are only two sympatric adaptive radiations of trophic specialists across this range (Martin and Wainwright 2011). One radiation is endemic to San Salvador Island, Bahamas, containing a generalist algivorous and detritivorous species, Cyprinodon variegatus, and two trophic specialist species, a molluscivore C. brontotheroides and a scale-eater C. desquamator (Martin and Wainwright, 2011; Martin and Wainwright, 2013; Richards and Martin, 2017). Scale-eating and molluscivore niches are uniquely derived within this sympatric radiation on San Salvador Island relative to generalist outgroup populations spread across the Caribbean and desert interior of North America (Martin and Feinstein 2014; Richards and Martin 2016). These two specialist species diverged from a generalist common ancestor within the past $10 \mathrm{kya}$, drawing adaptive alleles from ancient standing genetic variation across the Caribbean (Richards et al. 2020; McGirr and Martin 2020), whereas the most divergent generalist population in our study, the checkered pupfish Cualac tessellatus, has persisted for up to 5 Mya in El Potosí desert spring system in Mexico (Echelle et al. 2005). Thus, this radiation provides an excellent opportunity to compare microbiome divergence within a sympatric adaptive radiation of trophic specialists to closely related and ancient outgroup generalist taxa which have not substantially shifted their dietary niches.

We compared gut length, overall microbiome diversity, and enrichment of specific microbial taxa among three sympatric Cyprinodon pupfish species from two different isolated lake populations on San Salvador Island, Bahamas to three generalist species: closely related C. laciniatus from Lake Cunningham, New Providence Island, Bahamas; more distantly related C. variegatus from Fort Fisher, North Carolina; and the most closely related extant genus Cualac tessellatus from San Luis Potosí, Mexico. We raised all these species in a common laboratory environment for at least one generation and fed them an identical commercial pellet diet for one month before sampling gut microbiomes. We addressed the following questions: 1) Do microbial gut communities vary by diet or phylogenetic distance among these species? 2) Is there a microbiome signal associated with lepidophagy (scale-eating) or molluscivory?

\section{Materials and Methods}

\section{Sampling and preparation of gut microbiome samples}

Colonies of Cyprinodon pupfishes were collected from two hypersaline lakes on San Salvador Island, Bahamas (Crescent Pond and Osprey Lake) and Lake Cunningham, Bahamas in March, 2018 and were reared in aquaria at the University of California, Berkeley. Additional generalist populations were collected in May, 2018 from Fort Fisher Estuary in North Carolina. Cualac tessellatus eggs were provided by the Zoological Society of London and reared in the lab to produce a large second generation used for the four samples in this study. All samples, except for the recently collected NC population, came from first or second-generation captive-bred individuals reared in aquaria (40-80 L) according to species and location at 5-10 ppt salinity (Instant Ocean synthetic sea salt) and between 23 to $30^{\circ} \mathrm{C}$. Individuals used for this study were first fed once daily ad libitum with a single commercial pellet food (New Life Spectrum Cichlid Formula, New Life International, Inc., Homestead, FL), containing $34 \%$ crude protein, $5 \%$ crude fat, and $5 \%$ crude fiber, for one month without exposure to any other food or tankmates. All animal care and experiments were conducted under approved protocols and guidelines of the University of California, Berkeley Institutional Animal Care and Use Committee (AUP-2018-08-11373).

In total, forty fishes were euthanized in an overdose of MS-222 and the entire intestinal tissue was immediately 
excised (Cyprinodontidae do not possess stomachs; Wilson and Castro, 2010) for DNA extraction. Standard length and gut length were measured for all samples (Table $\mathrm{S} 1)$. Five individuals $\left(\mathrm{F}_{2}\right.$ generation) from each of three species (C. variegatus, C. brontotheroides, and C. desquamator) in both lake populations from San Salvador Island were sampled $(n=30$ total). In addition, we included the following pupfish species as outgroups to our study: C. laciniatus (F1 generation; Lake Cunningham, New Providence Island, Bahamas; $n=4$ ), C. variegatus (F0 generation; Fort Fisher, North Carolina, United States; $n=2$ ) plus liver tissue as a tissue control, and Cualac tessellatus (long-term captive colony; San Luis Potosí, Mexico, $n=4$ ).

Each gut was divided into proximal and distal regions for all San Salvador Island samples to compare microbial composition between these regions. All outgroup samples used whole intestines. In addition, the microbial community was isolated from aquaria water in two tanks which contained F2 individuals of Osprey Lake C. variegatus and Crescent Pond C. variegatus, and used as controls $(n=2)$. The Vincent J. Coates Genomics Sequencing Laboratory at the University of California, Berkeley also generated three controls, including a positive control and two no template controls (NTC). Microbial DNA extractions were performed in batches (stored on ice) immediately after intestinal dissections with the Zymobiomics DNA Miniprep Kit (Zymo Research, Irvine, CA).

$16 S$ amplicon sequencing of gut microbiomes

All extracted microbiome DNA samples were quantified with a Nanodrop ND-1000 spectrophotometer (range 4.2-474.9 ng/ $\mathrm{ll}$ ). All samples were then sent to the QB3 Vincent J. Coates Genomics Sequencing Laboratory at the University of California, Berkeley for automated library preparation and sequencing of $16 \mathrm{~S}$ rRNA amplicons using an Illumina Mi-Seq v3 (600 cycle). As part of the QB3 library preparation, the Forward ITS1 (ITS1f) - CTTGGTCATTTAGAGGAAGTAA and Reverse ITS1 (ITS2) - GCTGGGTTCTTCATCGATGC primers (Smith and Peay, 2014) were used for DNA metabarcoding markers for fungi (Smith and Peay, 2014). QB3 also used the following 16S rRNA primers for amplification of prokaryotes (archaea and bacteria): Forward 16S v4 (515Fb) - GTGYCAGCMGCCGCGGTAA, and Reverse 16S v4 (806Rb) - GGACTACNVGGGTWTCTAAT (Caporaso et al., 2011; Apprill et al. 2015).

\section{Bioinformatic Analysis / Quantification and Microbial Ecology Assessment of Samples}

All 16S rRNA amplicon sequences were processed through QIIME 2.0 (Bolyen et al. 2018) to identify microbe species and estimate abundances. Sequences from all 78 microbiome preps were imported into QIIME v. 2019.10.0. We determined there were no differences between proximal and distal regions of the gut for the San Salvador Island individuals, therefore we concatenated the Crescent Pond and Osprey Lake samples into one file, in which we had 48 samples which included experimental controls and quality controls from the QB3 facility (Table S2). There was no difference between the means of microbe counts in the foregut and the hindgut (paired t-test, $P=0.29$ ).

We used DADA2 (Callahan et al. 2016) for modeling and correcting Illumina-sequenced amplicon errors, removing chimeras, trimming low quality bases, and merging of forward and reverse reads using the following parameters: -p-trunc-len-f 270 -p-trunc-len-r 210. We used the QIIME alignment mafft software to align sequencesalignment mask to filter non-conserved and highly gapped columns from the aligned 16S sequences (Stackebrandt and Goodfellow, 1991). Next, we used qiime phylogeny midpoint-root to root the phylogeny of our $16 \mathrm{~S}$ amplicon sequences. Finally, we used qiime diversity alpha-rarefaction on all samples and we set the -p-max-depth to 10,000. We removed samples with 5,000 or less from our analyses.

We compared the beta diversity (qiime emperor plot ) of proximal and distal gut microbiomes of the San Salvador samples with a two-tailed paired $t$-test and found no significant differences between proximal and distal regions of the gut microbiome $(P=0.29)$. Therefore, we merged the proximal and distal samples for each individual from San Salvador Island, resulting in 48 samples. We also removed oneCualac tessellatus sample because of low read count (129 reads; Figure S1).

We used the classifier Silva 132 99\% 515F/806R (silva-132-99-515-806-nb-classifier) for training in identification of taxa from our samples. Afterwards the following files generated in QIIME were used in R (v. 
4.0.0) for further statistical analyses: table.qza, rooted-tree.qza, taxonomy.qza, and sample-metadata.tsv. We used the following R packages for further analyses: phyloseq (McMurdie and Holmes 2013) and ggplot2 (Wickham, 2016) with the following functions:distance, plot_bar, plot_ordination, andplot_richness . Before conducting any analyses, we removed the following taxa from our analyses, uncharacterized and Opisthokonta (eukaryotic sequences mainly due to fish $16 \mathrm{~S}$ amplicons). We estimated alpha diversity by using the plot_richness function and the Chao1 and Shannon's diversity indices. For beta diversity, we used theplot_ordination function and non-metric multidimensional scaling (NMDS) based on Bray-Curtis distances among samples. Hierarchical clustering was generated with the distance function along with hclust as part of fastcluster (Müllner, 2013) using the average linkage clustering method. The plot_bar function in the phyloseq package was used to visualize relative abundance of taxa. In our taxa plots we removed abundance counts of less than 400 from our analyses. We used ggplot2 to generate all figures (Wickham, 2016). Lastly, we used the Linear discriminant analysis Effect Size (LEfSe version 1.0; Segata et al. 2011) algorithm to identify microbial taxa that were significantly enriched in each of our specialists (scale-eater and molluscivore) in comparison to all other samples. This analysis was used to determine the features (i.e. organisms, clades, operational taxonomic units) to explain differences in assigned metadata categories. We used the nonparametric factorial Kruskal-Wallis rank-sum test to detect taxa with significant differential abundances between specialist samples and all generalist samples (scale-eaters versus generalists + molluscivores, molluscivores versus generalists + scale-eaters). We then used a Wilcoxon test for all pairwise comparisons between taxa within each significantly enriched class to compare to the class level. From the standard and gut length measurements, we used ANCOVA in R (v. 4.0.0) to test whether there was a significant difference among species based on gut length and standard length.

Lastly, we used generalized linear models (GLMs) in R to test the effects of diet (generalist, scale-eater, molluscivore), the fixed effect of location (Osprey Lake, San Salvador Island; Crescent Pond, San Salvador Island; Lake Cunningham, New Providence Island; Fort Fisher, North Carolina; and San Luis Potosí, Mexico), and their interaction on the response variables of principal coordinates axes 1 and 2 .

\section{Results}

\section{Intestinal lengths among species did not vary}

There was no significant difference in gut lengths among the species sampled (Figure S2; ANCOVA with covariate of log-transformed SL; $\left.F_{5,33}=0.916, P=0.483\right)$.

\section{Gut microbiome diversity and divergence among taxa}

We sequenced a total of 11,152,147 reads across all samples (Table S2). We identified 5,174 bacterial taxa in 48 samples. Similar to other ray-finned fishes (Youngblut et al. 2019), proteobacteria is the predominate microbial taxon (Figure S3). We did not find any significant differences among species in Chao1 or Shannon diversity indices (Kruskal-Wallace [pairwise], $P>0.05$; Figure 1). San Salvador Island pupfishes clustered together relative to the three outgroup generalist species, indicating strong host phylogenetic signal associated with overall microbiome diversity (Figure 2; Figure S4). Water and tissue controls were scattered throughout the NMDS plots but were clearly distinct from Cyprinodon microbiome samples with the exception of one tissue control that clustered near the outgroup species, possibly due to contamination during dissections (Figure 2).

Multiple regression analyses of the effects of dietary specialization (generalist, scale-eater, or molluscivore) and the fixed effect of population origin (two different lakes on San Salvador Island, Lake Cunningham, North Carolina, and El Potosí) on NMDS axes 1 and 2 confirmed that population origin and scale-eating had a significant effect on microbiome divergence along both axes (NMDS1: scale-eaters $P=0.001$; NMDS2: scale-eaters $P=0.018)$.

\section{Linear discriminate analyses of trophic specialist microbiota}

We found that an excess of taxa in the family Burkholderiaceaebest discriminated all lab-reared scale-eater individuals in two different lake populations from all other gut microbiome samples (Figures 3-4; linear 
discriminant analysis $\log$ score $=4.85$ ). In addition, we found a deficiency of Vibrionales, Vibrionaceae, and Vibrio in these scale-eater individuals relative to all other gut samples (LDA log scores $=-5.22,-5.22$, and -5.08 , respectively; Figure 4). Similarly, we found an excess of taxa in the family Rhodobacteraceae and class Planctomycetacia in the molluscivores relative to all other gut samples (Figure 5; LDA log scores of 4.39 and 4.37, respectively).

\section{Discussion}

Using a common garden experiment we show that differences in gut microbial diversity across Cyprinodon pupfish species largely reflect phylogenetic distance among generalist populations in support of phylosymbiosis (Bordenstein and Theis 2015), rather than novel trophic specializations as predicted by adaptive radiation theory. Our study is highly consistent with Ren et al. (2016) which also found limited microbiome divergence and minimal associations with ecomorph in an adaptive radiation of Puerto Rican Anolis lizards, even within wild lizards. Gut microbiome diversity has also been found to associate more strongly with geography than phylogeny (Godoy-Vitorino et al., 2012) or a combination of geography, diet, and host phylogeny (Antonopoulou et al., 2019). These emerging studies of microbiome divergence within adaptive radiations of hosts provide an important counterpoint to the classic expectation of rapid phenotypic diversification and speciation during adaptive radiation (Schluter 2000; Stroud and Losos 2016; Martin and Richards 2019; Gillespie et al. 2020).

A major caveat is that we did not examine the microbiota of wild-collected animals feeding on their diverse natural resources of macroalgae, scales, and snails. Scales form up to $50 \%$ of the diet in scale-eaters (Martin and Wainwright 2013) and wild gut microbiome samples surely would have revealed more substantial differences in microbiome diversity and composition among generalist and specialist species on San Salvador Island. However, our goal with this common garden study using lab-reared animals fed an identical generalist-type diet for one month was to uncover any genetically based microbiome differences in these taxa by eliminating environmental effects as much as possible. Pupfishes exhibit no parental care and deposit external eggs on the substrate so vertical transmission also appears highly unlikely (but see Satoh et al. 2019 for a potential example of vertical transmission in a scale-eating cichlid). Furthermore, by including two labreared colonies of each generalist and specialist species on San Salvador from genetically differentiated and ecologically divergent lake populations (Martin et al. 2016; Richards and Martin 2017), we aimed to connect significant differences in microbiome composition observed in our specialist species to their specialized diets, rather than their lake environment or genetic background. This provides strong evidence of genetic divergence in the host associated with trophic specialization. These results are all the more surprising because trophic specialists show very little genetic differentiation from generalists $\left(F_{s t}=0.1-0.3\right.$; Martin and Feinstein 2014; Richards et al. 2020). Indeed, there are only a few thousand nearly fixed or fixed SNPs $\left(F_{s t}>0.95\right)$ between scale-eaters and molluscivores out of over 10 million segregating SNPs and as few as 157 fixed SNPs and 87 deletions in scale-eaters (McGirr and Martin 2020). However, this minimal set of genetic differences may be driving differences in gut microbiome composition. Intriguingly, the only fixed coding indel uncovered so far in this system is a fixed deletion in all scale-eater populations of the fifth exon of the gene gpa33 (McGirr and Martin 2020). This gene is expressed exclusively in the intestinal epithelium and mice knockouts display a range of inflammatory intestinal pathologies in mice (Williams et al. 2015), suggesting it may play a role in shifting the gut microbiota of scale-eaters that we observed in this study. Overall, metabolic processes were the single most enriched category among all differentially expressed genes between these trophic specialists at the $8 \mathrm{dpf}$ larval stage, accounting for $20 \%$ of differential expression (McGirr and Martin 2018).

\section{Adaptive microbiota in scale-eating pupfish}

Fish scales are composed of a deep layer that is mostly collagen type I (Harikrishna et al. 2017); therefore, we predicted that any adaptive microbes within the scale-eater gut would have collagen degrading properties. This includes Bacillus, Clostridium, and Vibrio taxa, which are well-known for microbial collagenase enzymes (Duarte et al. 2016). We found a significant reduction of Vibrio taxa within the scale-eater gut from both lake populations (Figs. 3-4). Although it is not clear why there are fewer taxa, the significant shift in a major collagenase-producing group suggests the potential for an adaptive scale-eater microbiome, even in the 
absence of dietary scales (except perhaps incidental aggression and ingestion of scales among tankmates). We also found significant enrichment of the family Burkholderiaceae in both scale-eater populations (Figs. 3-4). Burkholderiaceae is a family of proteobacteria which contains many human and animal pathogens (diCenzo et al. 2019), plant and insect symbionts (Gyaneshwar et al. 2011; Takeshita and Kikuchi 2017), and can be found in soil, water, and polluted environments (Coenye and Vandamme 2003; Estrada-de los Santos et al. 2016). They also include some collagenase-producing bacteria, such as Burkholderia pseudomallei (UniProtKB - A3P3M6; Rainbow et al. 2004), which is the causative agent of melioidosis in humans (Holden et al. 2004).

In contrast to a microbiome study of the adaptive radiation of Tanganyikan cichlids (Baldo et al. 2015), we found no evidence of Clostridia enrichment in scale-eaters nor a reduction of microbial diversity in this carnivorous species. This may be due to the very young $10 \mathrm{kya}$ age of the scale-eating pupfish relative to the comparatively ancient 12 Mya Tanganyikan radiation and Perissodus scale-eating clade (Koblmueller et al. 2007; Martin and Wainwright 2013).

\section{Nonadaptive microbiota in molluscivore pupfish}

We found enrichment of the families Rhodobacteraceae andPlanctomycetacia within the molluscivore gut from both lake populations (Figure 5). However, these families have no clear role in anything related to mollusc digestion or even increased levels of protein, lipids, or chitin in the diet (due to some molluscivores specializing on ostracods during periods of abundance). Taxa from these taxonomic group are known to be found within aquatic environments (Simon et al. 2017; Yilmaz et al. 2016). Marine Rhodobacteraceae have a key role in biogeochemical cycling, make up about $30 \%$ of bacterial communities in the pelagic environment, and generally have a mutualistic relationship with eukaryotes providing vitamins to these groups (Simon et al. 2017). Both families are known for aquatic cellulose-decomposing taxa (Ring $\varnothing$ et al. 2016; Kim et al. 2016), which suggests this microbiome shift may help more with macroalgae digestion rather than molluscs, despite previous observations that macroalgae forms the largest component of the generalist pupfish diet in the hypersaline lakes of San Salvador Island, Bahamas (Martin and Wainwright 2013).

\section{Conclusion}

Many studies have focused on understanding digestion and assimilation within a variety of vertebrates and invertebrates, but there is limited information about the cooperative process between the host intestine cells and gut microbiota, and their role in eco-evolutionary dynamics during rapid species diversification (German et al. 2015; Terra et al. 2019; Baldo et al. 2017). We found evidence for a genetically-based adaptive shift in the scale-eater microbiome, even when hosts were reared in identical environments on identical non-scale diets. However, it is still unknown to what extent this microbiome shift will improve digestion of the collagen found in scales, for example, as demonstrated for the gut fauna in the scale-eating khavalchor catfish (Gosavi et al. 2018). Despite unique and highly specialized pupfish dietary adaptations within shared hypersaline lake habitats, overall gut microbial diversity did not follow the expected pattern of rapid diversification and divergence as observed in their hosts, calling into question how eco-evolutionary dynamics between host and symbiont proceed during adaptive radiation.

\section{Acknowledgements}

This research was supported by NSF CAREER Award 1938571 and NIH/NIDCR R01 DE027052 grants to CHM. We thank L. Smith in the Evolutionary Genetics Lab at the University of California, Berkeley, for generous logistical assistance in preparing microbiome samples; S. McDevitt, C. Miller, and D. Pappas at the Vincent J. Coates Genomics Sequencing Laboratory California Institute for Quantitative Biosciences (QB3) for processing our microbiome samples for $16 \mathrm{~S}$ amplicon sequencing; R. Berlemont at California State University, Long Beach and C. Weihe from the Microbiome Consortium at the University of California, Irvine for suggestions on microbiome extraction protocols and bioinformatic workflow. We thank the Zoological Society of London for providing C. tessellatus eggs and the governments of the Bahamas and United States for permission to collect and export Cyprinodonsamples. 


\section{References}

Apprill, A., McNally, S., Parsons, R., \& Weber, L. (2015). Minor revision to V4 region SSU rRNA 806R gene primer greatly increases detection of SAR11 bacterioplankton. Aquatic Microbial Ecology , 75(2), 129-137.

Antonopoulou, E., Nikouli, E., Piccolo, G., Gasco, L., Gai, F., Chatzifotis, S., .. \& Kormas, K. A. (2019). Reshaping gut bacterial communities after dietary Tenebrio molitor larvae meal supplementation in three fish species. Aquaculture , 503, 628-635.

Baldo, L., Riera, J. L., Tooming-Klunderud, A., Albà, M. M., \& Salzburger, W. (2015). Gut microbiota dynamics during dietary shift in eastern African cichlid fishes. PloS one, 10(5), e0127462.

Baldo, L., Pretus, J. L., Riera, J. L., Musilova, Z., Nyom, A. R. B., \& Salzburger, W. (2017). Convergence of gut microbiotas in the adaptive radiations of African cichlid fishes. The ISME journal , 11(9), 1975.

Baldo, L., Riera, J. L., Salzburger, W., \& Barluenga, M. (2019). Phylogeography and ecological niche shape the cichlid fish gut microbiota in Central American and African lakes. Frontiers in microbiology, 10, 2372.

Bolyen, E., Rideout, J. R., Dillon, M. R., Bokulich, N. A., Abnet, C. C., Al-Ghalith, G. A., ... \& Bai, Y. (2019). Reproducible, interactive, scalable and extensible microbiome data science using QIIME 2. Nature biotechnology, 37 (8), 852-857.

Bordenstein, S. R., \& Theis, K. R. (2015). Host biology in light of the microbiome: ten principles of holobionts and hologenomes. PLoS Biol, 13 (8), e1002226.

Brooks, A. W., Kohl, K. D., Brucker, R. M., van Opstal, E. J., \& Bordenstein, S. R. (2016). Phylosymbiosis: relationships and functional effects of microbial communities across host evolutionary history. PLoS biology , 14 (11), e2000225.

Callahan, B. J., McMurdie, P. J., Rosen, M. J., Han, A. W., Johnson, A. J. A., \& Holmes, S. P. (2016). DADA2: high-resolution sample inference from Illumina amplicon data. Nature methods , 13(7), 581.

Caporaso, J. G., Lauber, C. L., Walters, W. A., Berg-Lyons, D., Lozupone, C. A., Turnbaugh, P. J., \& Knight, R. (2011). Global patterns of $16 \mathrm{~S}$ rRNA diversity at a depth of millions of sequences per sample.Proceedings of the national academy of sciences, 108(Supplement 1), 4516-4522.

Coenye, T., \& Vandamme, P. (2003). Diversity and significance of Burkholderia species occupying diverse ecological niches.Environmental microbiology , 5(9), 719-729.

diCenzo, G. C., Mengoni, A., \& Perrin, E. (2019). Chromids aid genome expansion and functional diversification in the family Burkholderiaceae. Molecular biology and evolution, 36(3), 562-574.

Duarte, A. S., Correia, A., \& Esteves, A. C. (2016). Bacterial collagenases-a review. Critical Reviews in Microbiology , 42(1), 106-126.

Echelle, A. A., \& Echelle, A. F. (1992). Mode and pattern of speciation in the evolution of inland pupfishes of the Cyprinodon variegatuscomplex (Teleostei: Cyprinodontidae): an ancestor-descendent hypothesis.Systematics, historical ecology, and North American freshwater fishes (ed. by R.L. Mayden), pp. 691-709. Stanford University Press, Stanford, CA.

Estrada-De Los Santos, P., Rojas-Rojas, F. U., Tapia-García, E. Y., Vásquez-Murrieta, M. S., \& Hirsch, A. M. (2016). To split or not to split: an opinion on dividing the genus Burkholderia .Annals of Microbiology , 66(3), 1303-1314.

German, D. P., Sung, A., Jhaveri, P., \& Agnihotri, R. (2015). More than one way to be an herbivore: convergent evolution of herbivory using different digestive strategies in prickleback fishes (Stichaeidae). Zoology , 118 (3), 161-170.

Gillespie, R. G., Bennett, G. M., De Meester, L., Feder, J. L., Fleischer, R. C., Harmon, L. J., .. \& Parent, C. E. (2020). Comparing adaptive radiations across space, time, and taxa. Journal of Heredity, 111(1), 1-20. 
Godoy-Vitorino, F., Leal, S. J., Díaz, W. A., Rosales, J., Goldfarb, K. C., García-Amado, M. A., ... \& Domínguez-Bello, M. G. (2012). Differences in crop bacterial community structure between hoatzins from different geographical locations. Research in Microbiology , 163(3), 211-220.

Gosavi, S. M., Kharat, S. S., Kumkar, P., \& Navarange, S. S. (2018). Interplay between behavior, morphology and physiology supports lepidophagy in the catfish Pachypterus khavalchor (Siluriformes: Horabagridae). Zoology, 126, 185-191.

Gould, A. L., Zhang, V., Lamberti, L., Jones, E. W., Obadia, B., Korasidis, N., .. \& Ludington, W. B. (2018). Microbiome interactions shape host fitness. Proceedings of the National Academy of Sciences , 115 (51), E11951-E11960.

Gyaneshwar, P., Hirsch, A. M., Moulin, L., Chen, W. M., Elliott, G. N., Bontemps, C., ... \& Young, J. P. W. (2011). Legume-nodulating betaproteobacteria: diversity, host range, and future prospects. Molecular plant-microbe interactions, 24(11), 1276-1288.

Harikrishna, N., Mahalakshmi, S., Kumar, K. K., \& Reddy, G. (2017). Fish Scales as Potential Substrate for Production of Alkaline Protease and Amino Acid Rich Aqua Hydrolyzate by Bacillus altitudinisGVC11. Indian journal of microbiology, 57(3), 339-343.

Holden, M. T., Titball, R. W., Peacock, S. J., Cerdeño-Tárraga, A. M., Atkins, T., Crossman, L. C., ... \& Sebaihia, M. (2004). Genomic plasticity of the causative agent of melioidosis, Burkholderia pseudomallei. Proceedings of the National Academy of Sciences, 101(39), 14240-14245.

Kim, J. W., Brawley, S. H., Prochnik, S., Chovatia, M., Grimwood, J., Jenkins, J., .. \& \& Schmutz, J. (2016). Genome analysis of Planctomycetes inhabiting blades of the red alga Porphyra umbilicalis. PLoS One, 11(3), e0151883.

Koblmüller, S., Egger, B., Sturmbauer, C., \& Sefc, K. M. (2007). Evolutionary history of Lake Tanganyika's scale-eating cichlid fishes. Molecular phylogenetics and evolution, 44(3), 1295-1305.

Lim, S. J., \& Bordenstein, S. R. (2020). An introduction to phylosymbiosis. Proceedings of the Royal Society $B$, 287 (1922), 20192900.

Losos, J. B. (2008). Phylogenetic niche conservatism, phylogenetic signal and the relationship between phylogenetic relatedness and ecological similarity among species. Ecology letters, 11(10), 995-1003.

Loo, W. T., García-Loor, J., Dudaniec, R. Y., Kleindorfer, S., \& Cavanaugh, C. M. (2019). Host phylogeny, diet, and habitat differentiate the gut microbiomes of Darwin's finches on Santa Cruz Island.Scientific Reports , 9(1), 1-12.

Macke, E., Tasiemski, A., Massol, F., Callens, M., \& Decaestecker, E. (2017). Life history and ecoevolutionary dynamics in light of the gut microbiota. Oikos , 126(4), 508-531.

Martin, C. H., Crawford, J. E., Turner, B. J., \& Simons, L. H. (2016). Diabolical survival in Death Valley: recent pupfish colonization, gene flow and genetic assimilation in the smallest species range on earth. Proceedings of the Royal Society B: Biological Sciences, 283(1823), 20152334.

Martin, C. H., \& Feinstein, L. C. (2014). Novel trophic niches drive variable progress towards ecological speciation within an adaptive radiation of pupfishes. Molecular Ecology, 23(7), 1846-1862.

Martin, C. H., \& Richards, E. J. (2019). The paradox behind the pattern of rapid adaptive radiation: how can the speciation process sustain itself through an early burst? Annual Review of Ecology, Evolution, and Systematics, 50, 569-593.

Martin, C. H., \& Wainwright, P. C. (2011). Trophic novelty is linked to exceptional rates of morphological diversification in two adaptive radiations of Cyprinodon pupfish. Evolution: International Journal of Organic Evolution, 65 (8), 2197-2212. 
Martin, C. H., \& Wainwright, P. C. (2013). On the measurement of ecological novelty: scale-eating pupfish are separated by 168 my from other scale-eating fishes. PLoS One, 8(8), e71164.

Matthews, B., Aebischer, T., Sullam, K. E., Lundsgaard-Hansen, B., \& Seehausen, O. (2016). Experimental evidence of an eco-evolutionary feedback during adaptive divergence. Current Biology, 26 (4), 483-489.

McGirr, J. A., \& Martin, C. H. (2018). Parallel evolution of gene expression between trophic specialists despite divergent genotypes and morphologies. Evolution letters, 2(2), 62-75.

McGirr, J. A., \& Martin, C. H. (2020). Few fixed variants between trophic specialist pupfish species reveal candidate cis-regulatory alleles underlying rapid craniofacial divergence. Molecular Biology and Evolution.

McMurdie, P. J., \& Holmes, S. (2013). phyloseq: an R package for reproducible interactive analysis and graphics of microbiome census data. PloS one , 8 (4), e61217.

Mullner, D. (2013). fastcluster: Fast hierarchical, agglomerative clustering routines for R and Python. Journal of Statistical Software, 53 (9), 1-18.

Olsson, K. H., Martin, C. H., \& Holzman, R. (2020). Hydrodynamic simulations of the performance landscape for suction-feeding fishes reveal multiple peaks for different prey types. Integrative and Comparative Biology .

Post, D. M., \& Palkovacs, E. P. (2009). Eco-evolutionary feedbacks in community and ecosystem ecology: interactions between the ecological theatre and the evolutionary play. Philosophical Transactions of the Royal Society B: Biological Sciences, 364(1523), 1629-1640.

Rainbow, L., Wilkinson, M. C., Sargent, P. J., Hart, C. A., \& Winstanley, C. (2004). Identification and expression of a Burkholderia pseudomallei collagenase in Escherichia coli. Current microbiology, 48(4), 300-304.

Ren, T., Kahrl, A. F., Wu, M., \& Cox, R. M. (2016). Does adaptive radiation of a host lineage promote ecological diversity of its bacterial communities? A test using gut microbiota of Anolis lizards.Molecular ecology , 25(19), 4793-4804.

Rennison, D. J., Rudman, S. M., \& Schluter, D. (2019). Parallel changes in gut microbiome composition and function during colonization, local adaptation and ecological speciation. Proceedings of the Royal Society $B$ , 286 (1916), 20191911.

Richards, E. J., \& Martin, C. H. (2017). Adaptive introgression from distant Caribbean islands contributed to the diversification of a microendemic adaptive radiation of trophic specialist pupfishes.PLoS genetics , 13(8), e1006919.

Richards, E. J., McGirr, J. A., Wang, J., St John, M. E., Poelstra, J. W., Solano, M. J., .. \& Martin, C. H. (2020). Major stages of vertebrate adaptive radiation are assembled from a disparate spatiotemporal landscape. bioRxiv.

Ringo, E., Zhou, Z., Vecino, J. G., Wadsworth, S., Romero, J., Krogdahl, A., .. \& Owen, M. (2016). Effect of dietary components on the gut microbiota of aquatic animals. A never-ending story? Aquaculture nutrition , 22(2), 219-282.

Rudman, S. M., Barbour, M. A., Csillery, K., Gienapp, P., Guillaume, F., Hairston Jr, N. G., .. \& \& Schmidt, P. S. (2018). What genomic data can reveal about eco-evolutionary dynamics. Nature ecology $\&$ evolution, 2(1), 9-15.

Rundell, R. J., \& Price, T. D. (2009). Adaptive radiation, nonadaptive radiation, ecological speciation and nonecological speciation. Trends in Ecology \& Evolution, 24(7), 394-399.

St. John, M. E., McGirr, J. A., \& Martin, C. H. (2019). The behavioral origins of novelty: did increased aggression lead to scale-eating in pupfishes? Behavioral Ecology, 30 (2), 557-569. 
Satoh, S., Awata, S., Tanaka, H., Jordan, L. A., Kakuda, U., Hori, M., \& Kohda, M. (2019). Bi-parental mucus provisioning in the scale-eating cichlid Perissodus microlepis (Cichlidae). Biological Journal of the Linnean Society, 128(4), 926-935.

Schluter, D. (2000). The ecology of adaptive radiation. OUP Oxford.

Segata, N., Izard, J., Waldron, L., Gevers, D., Miropolsky, L., Garrett, W. S., \& Huttenhower, C. (2011). Metagenomic biomarker discovery and explanation. Genome biology , 12(6), 1-18.

Simon, M., Scheuner, C., Meier-Kolthoff, J. P., Brinkhoff, T., Wagner-Dobler, I., Ulbrich, M., .. \& Goker, M. (2017). Phylogenomics of Rhodobacteraceae reveals evolutionary adaptation to marine and non-marine habitats. The ISME journal, 11(6), 1483-1499.

Smith, D. P., \& Peay, K. G. (2014). Sequence depth, not PCR replication, improves ecological inference from next generation DNA sequencing. PloS one , 9 (2), e90234.

Stackebrandt, E., \& Goodfellow, M. (1991). Nucleic acid techniques in bacterial systematics . Wiley.

Stroud, J. T., \& Losos, J. B. (2016). Ecological opportunity and adaptive radiation. Annual Review of Ecology, Evolution, and Systematics , 47.

Takeshita, K., \& Kikuchi, Y. (2017). Riptortus pedestris and Burkholderia symbiont: an ideal model system for insect-microbe symbiotic associations. Research in Microbiology, 168(3), 175-187.

Terra, W. R., Barroso, I. G., Dias, R. O., \& Ferreira, C. (2019). Molecular physiology of insect midgut. Advances in Insect Physiology , $56,117$.

Trevelline, B. K., \& Kohl, K. D. (2020). Microbial control over host diet selection. bioRxiv.

Turcotte, M. M., Reznick, D. N., \& Daniel Hare, J. (2013). Experimental test of an eco-evolutionary dynamic feedback loop between evolution and population density in the green peach aphid. The American Naturalist , 181 (S1), S46-S57.

Walters, A. W., Hughes, R. C., Call, T. B., Walker, C. J., Wilcox, H., Petersen, S. C., .. \& \& Chaston, J. M. (2020). The microbiota influences the Drosophila melanogaster life history strategy. Molecular Ecology , $29(3), 639-653$.

Wickham, H. (2016). ggplot2: elegant graphics for data analysis. springer.

Williams, B. B., Tebbutt, N. C., Buchert, M., Putoczki, T. L., Doggett, K., Bao, S., .. \& \& Scott, A. M. (2015). Glycoprotein A33 deficiency: a new mouse model of impaired intestinal epithelial barrier function and inflammatory disease. Disease models \& mechanisms, 8(8), 805-815.

Wilson, J. M., \& Castro, L. F. C. (2010). Morphological diversity of the gastrointestinal tract in fishes. In Fish physiology (Vol. 30, pp. 1-55). Academic Press.

Yilmaz, P., Yarza, P., Rapp, J. Z., \& Glockner, F. O. (2016). Expanding the world of marine bacterial and archaeal clades. Frontiers in microbiology, 6, 1524.

Youngblut, N. D., Reischer, G. H., Walters, W., Schuster, N., Walzer, C., Stalder, G., .. \& Farnleitner, A. H. (2019). Host diet and evolutionary history explain different aspects of gut microbiome diversity among vertebrate clades. Nature communications , 10(1), 1-15.

\section{Data Accessibility}

Data will be deposited to Dryad and NCBI SRA.

\section{Author Contributions}

JH prepared all samples for sequencing, conducted statistical analyses, and wrote the manuscript. CHM revised the manuscript, acquired samples, and provided funding. Both authors designed the study. 
Figure 1
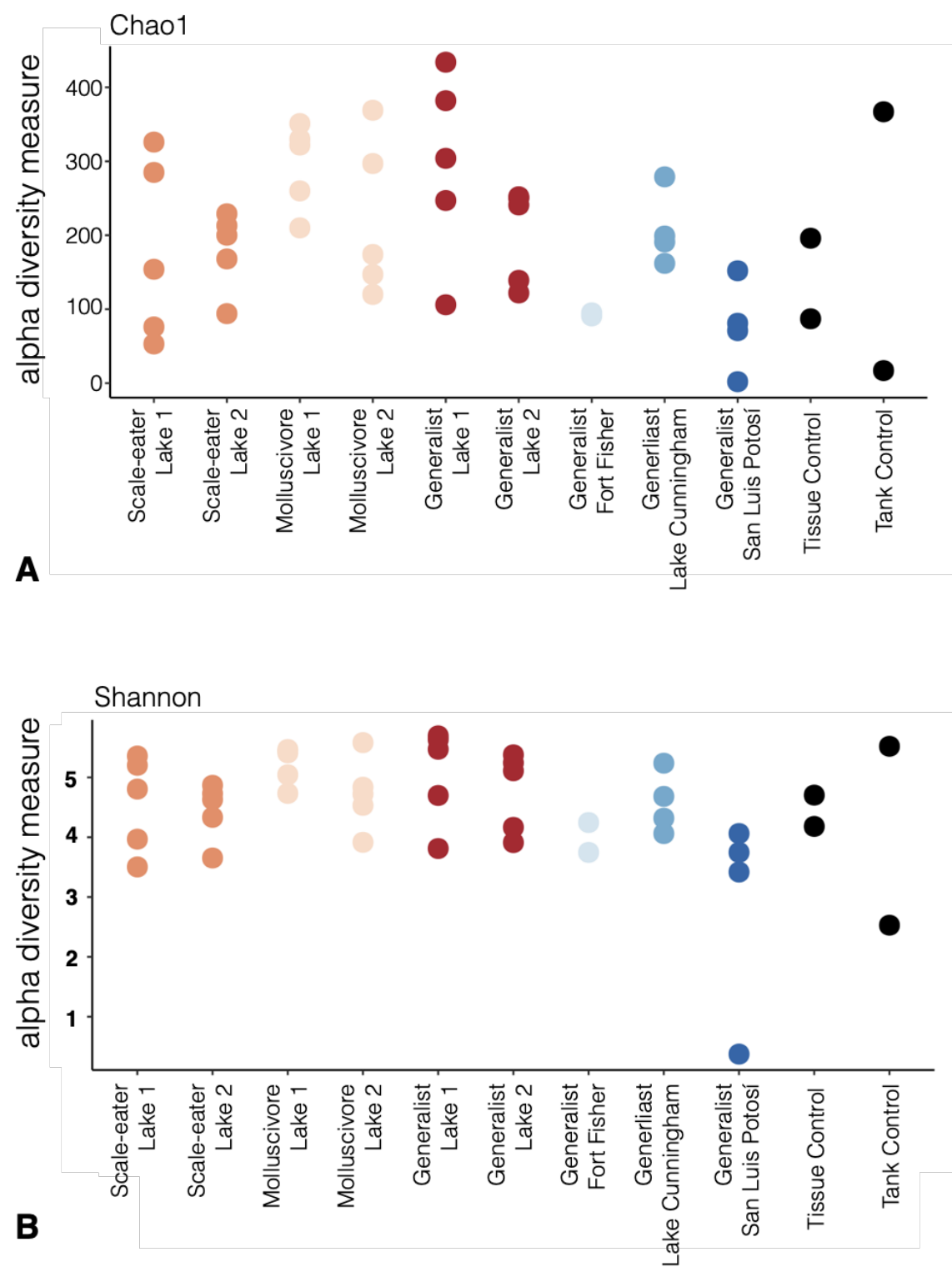

Figure 1: Alpha diversity of Cyprinodon pupfishes gut microbiomes based on parental location and diet type along with controls. Lake 1 indicates Crescent Pond and Lake 2 represents Osprey Lake, both located on San Salvador Island in the Bahamas. Alpha diversity is represented by (A) Chaol and (B) Shannon diversity for the estimate of species richness from gut microbiomes from all fishes in this study. 

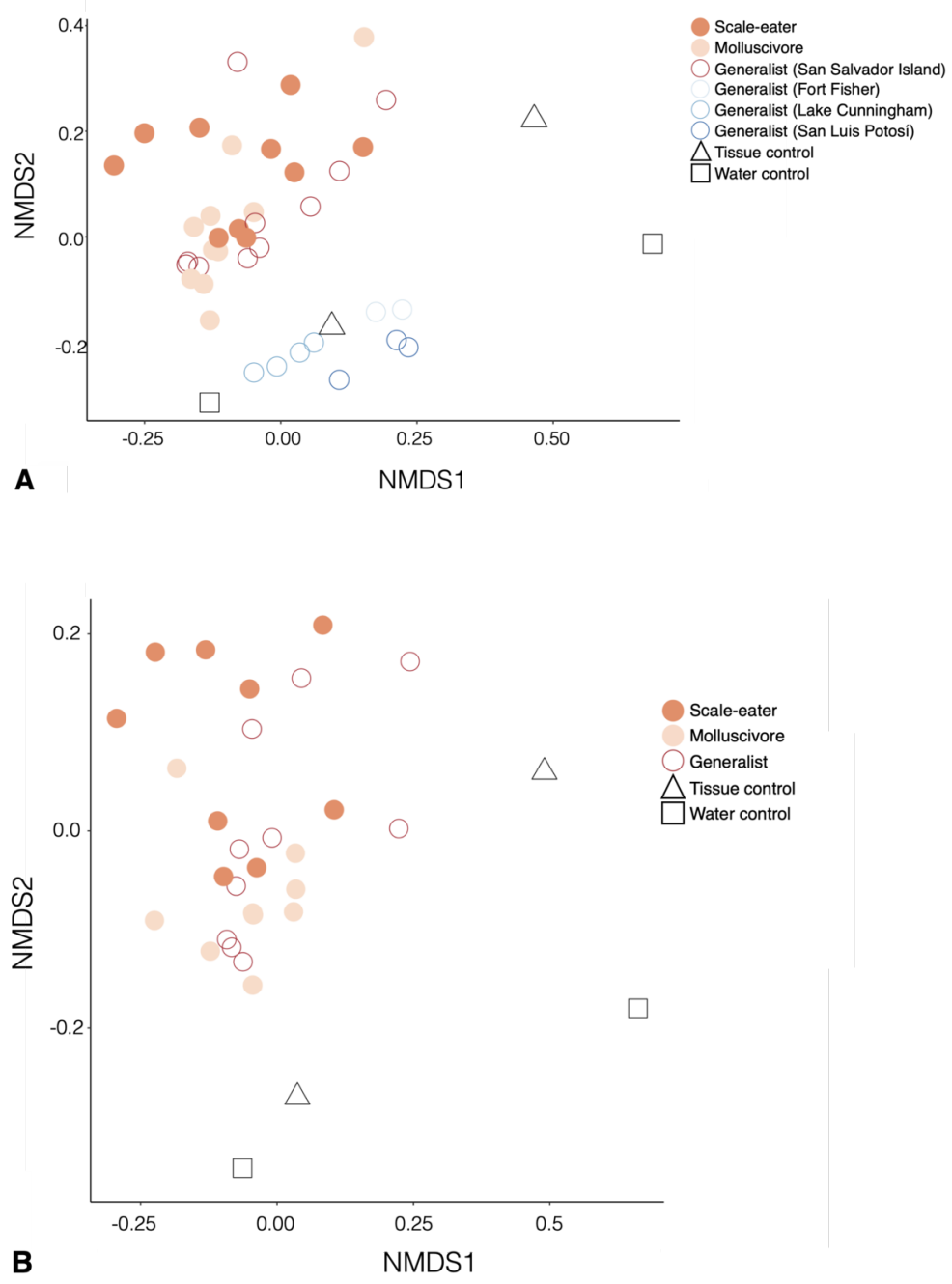

Figure 2: Non-metric multidimensional scaling (NMDS) plots of Cyprinodon pupfish gut microbiomes. A) NMDS plot based on allCyprinodon pupfish gut samples labeled according to species and diet including controls $(n=43)$. B) NMDS plot of the threeCyprinodon pupfish species (F2 generation) from San Salvador Island including controls $(n=34)$. Closed circles represent the two specialists (scale-eater and molluscivore) and open circles represent generalists. Open squares and triangles represent controls used in this study. 


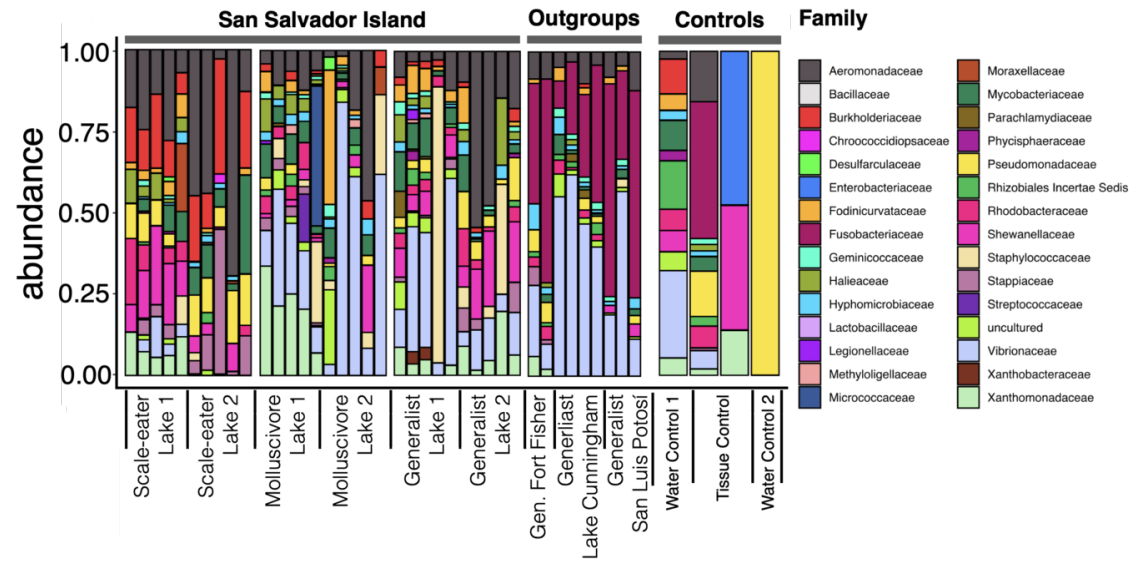

Figure 3: Taxa plot of the microbial composition of the Cyprinodon gut microbiome and controls. Bars show proportions (relative abundance) of taxa at the family level per individual gut microbiome. Lake 1 indicates Crescent Pond and Lake 2 represents Osprey Lake, both located on San Salvador Island in the Bahamas. Taxa which contained uncharacterized and Opisthokonta (eukaryotic sequences) were removed and taxa with a count of 400 or greater were represented. Taxa were grouped according to species and location (controls included). 


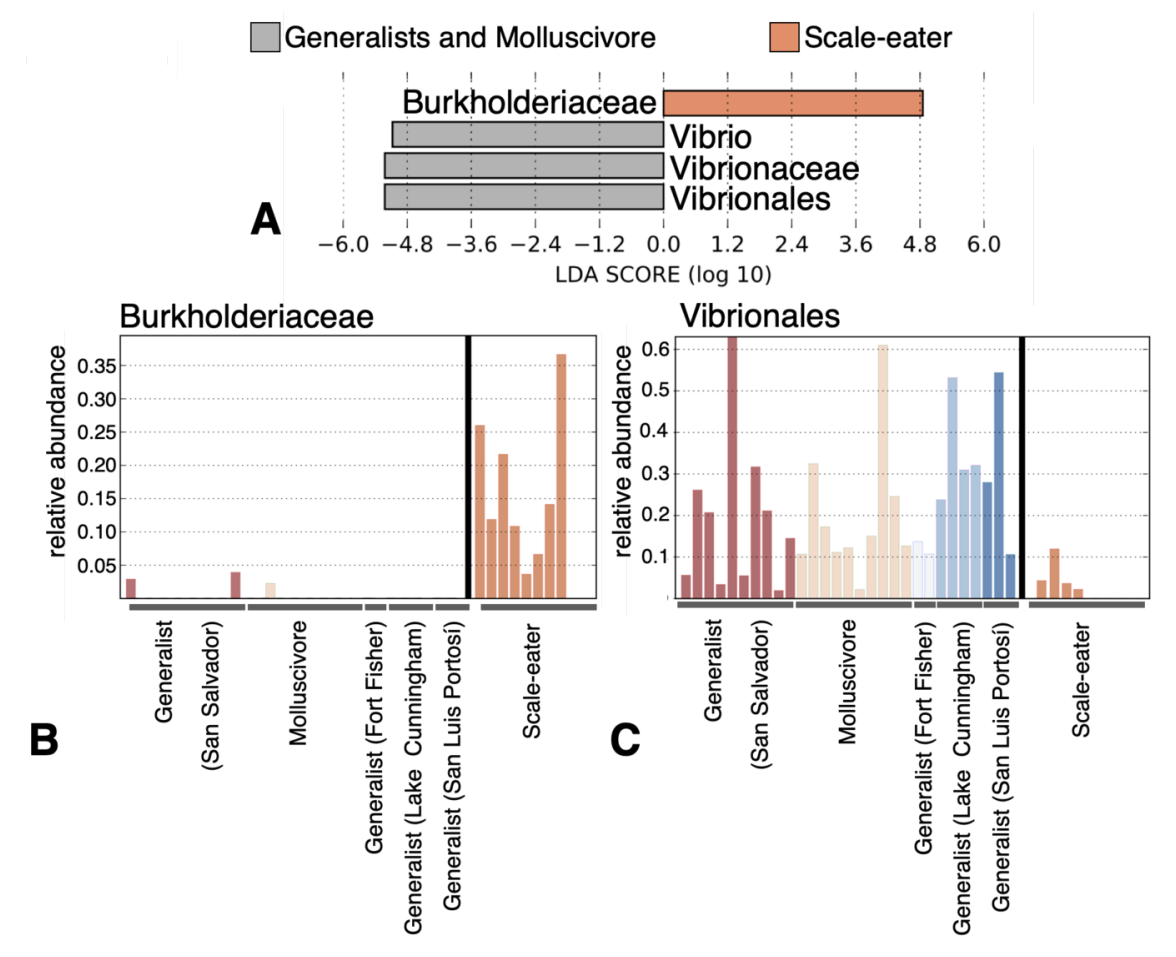

Figure 4: Linear discriminate analysis between Cyprinodon desquamator (scale-eater) and non-scale eaters. A) Log scores of the top four dominant loadings on LEfSe discriminate axis separating scale-eaters from all other pupfish samples. B) Relative abundance of the family Burkholderiaceae and the order C) Vibrionalesamong all pupfish gut microbiomes. 


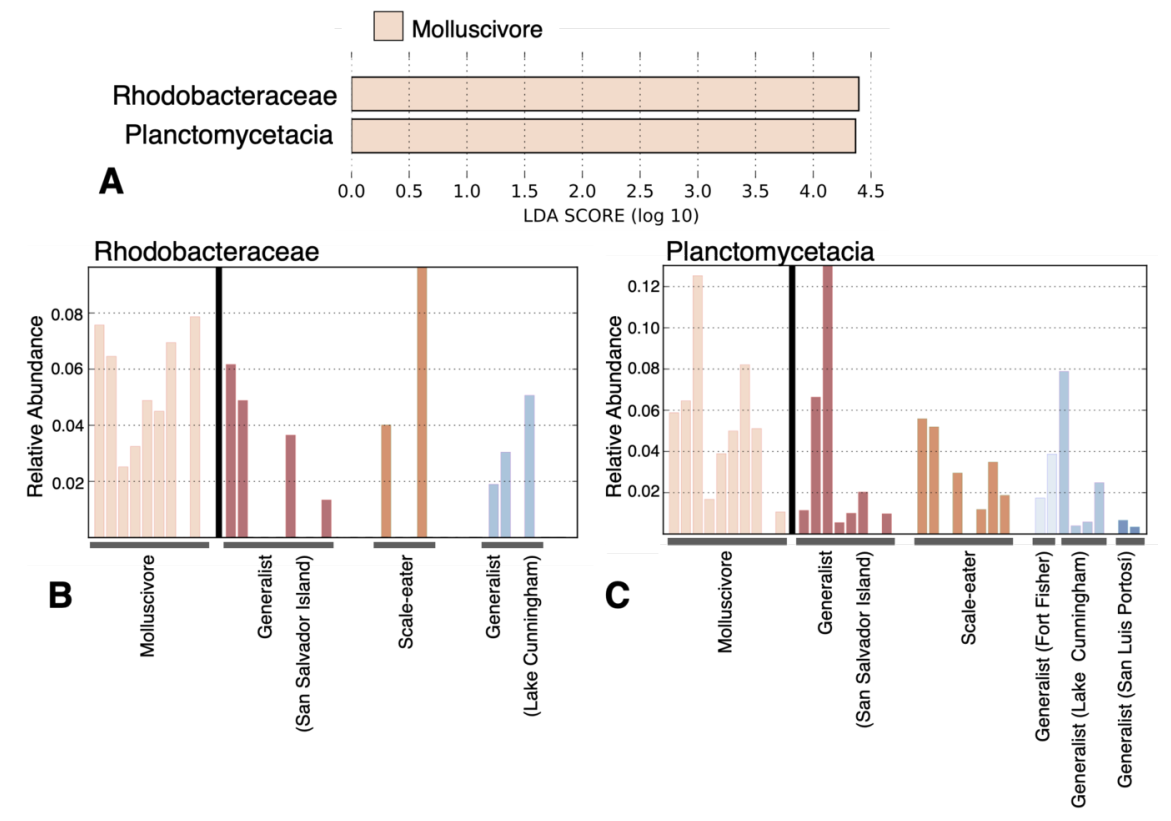

Figure 5: Linear discriminate analysis between Cyprinodon brontotheroides (molluscivore) and non-molluscivores. A) Log scores of the top two dominant loadings on the LEfSe discriminate axis separating molluscivores from all other pupfish samples. B) Relative abundance of the family Rhodobacteraceae and the class C) Planctomycetacia from all Cyprinodon gut microbiomes. 